\title{
Clinical efficacy of acetylcysteine combined with tetrandrine tablets in the treatment of silicosis and the effect on serum IL-6 and TNF- $\alpha$
}

\author{
JIANLING SUN, PINGPING SONG, YAN WANG and YANXIA CHEN \\ Department of Occupational Disease, Qingdao Central Hospital, Qingdao, Shandong 266042, P.R. China
}

Received June 4, 2019; Accepted July 30, 2019

DOI: $10.3892 /$ etm.2019.7966

\begin{abstract}
Clinical efficacy of acetylcysteine combined with tetrandrine tablets in the treatment of silicosis and the effect on serum levels of interleukin-6 (IL-6) and tumor necrosis factor- $\alpha(\mathrm{TNF}-\alpha)$ in patients with silicosis was investigated. One hundred and ninety-six patients with silicosis admitted to Qingdao Central Hospital, aged 40 to 70 years, were collected by prospective analysis. The patients were divided into two groups according to the different methods of treatment. The 108 patients in the control group received routine treatment, including anti-inflammatory, cough and asthma relief and 88 patients in the observation group were treated with tetrandrine combined with $\mathrm{N}$-acetylcysteine on the basis of routine treatment. The curative effect of the two groups was analyzed by X-ray, and the incidence of adverse reactions was compared between the two groups. There was no significant difference in the effective rate between the two groups $(\mathrm{P}>0.05)$. After treatment, respiratory rate $(\mathrm{R} R)$ decreased, and forced vital capacity (FVC), and first second forced expiratory volume (FEV1) increased in both groups $(\mathrm{P}<0.05)$. RR in the observation group was lower than that in the control group $(\mathrm{P}<0.05), \mathrm{FVC}$ and FEV1 were higher than those in the control group $(\mathrm{P}<0.05)$. There was no significant difference in serum IL- 6 and TNF- $\alpha$ levels between the two groups before treatment $(\mathrm{P}>0.05)$. After treatment, the levels of IL-6 and TNF- $\alpha$ in the two groups decreased $(\mathrm{P}<0.05)$, and the IL- 6 and TNF- $\alpha$ levels in the observation group after treatment were significantly lower than those in the control group $(\mathrm{P}<0.05)$. According to the effect of clinical treatment, the patients were re-divided into two groups. In conclusion, tetrandrine combined with acetylcysteine can effectively improve the clinical therapeutic effect of silicosis and alleviate the degree of inflammatory reaction in patients with
\end{abstract}

Correspondence to: Dr Yanxia Chen, Department of Occupational Disease, Qingdao Central Hospital, 127 Siliu South Road, Qingdao, Shandong 266042, P.R. China

E-mail: xjwd21@163.com

Key words: acetylcysteine, tetrandrine tablets, silicosis, drug combination, clinical effect, IL-6, TNF- $\alpha$ silicosis. The levels of IL-6 and TNF- $\alpha$ in peripheral blood are valuable for the clinical treatment of silicosis.

\section{Introduction}

Silicosis is the most serious occupational disease and is caused by long-term inhalation of large amount of free silica dust and deposition in the lung to cause pulmonary fibrosis as the main pathological change. Silicosis is the most common type of pneumoconiosis with rapid progression and poor prognosis (1-3). As its specific pathogenesis has not been fully studied, there are no specific drugs that can alleviate or reverse silica-induced lung injury. The purpose of clinical treatment is to control the progressive development of lung injury, improve the quality of life and prolong the life span of the patients $(4,5)$.

Tetrandrine is a calcium channel blocker, and can reduce the level of type I and III collagen mRNA in lung tissue and collagen deposition in the lung. It can effectively alleviate pulmonary fibrosis and inflammatory reaction of the body, and is an effective drug for the treatment of silicosis $(6,7)$. $\mathrm{N}$-acetylcysteine decreased the content of reactive oxygen species (ROS) in the lung tissue of rats exposed to silica, thus inhibiting apoptosis of mitochondria and alleviating the severity of pulmonary fibrosis (8). In recent years, a small number of studies have reported the therapeutic effect of tetrandrine combined with $\mathrm{N}$-acetylcysteine in silicosis. The combination of the two can effectively alleviate the clinical symptoms such as cough, chest tightness and improve respiratory function in patients with silicosis (9). However, this method has not been widely used in clinical treatment, and its therapeutic effect has not been widely confirmed. In a rat model of silicosis, the therapeutic effect of tetrandrine combined with $\mathrm{N}$-acetylcysteine was again verified. The results showed that the combined therapy could effectively reduce the levels of malondialdehyde (MDA), TNF- $\alpha$ and IL-6 in lung tissue and alleviate the silica-induced pulmonary fibrosis in rats (10). These studies have reported better therapeutic effects of tetrandrine combined with $\mathrm{N}$-acetylcysteine in silicosis.

In order to confirm the therapeutic effect of tetrandrine combined with $\mathrm{N}$-acetylcysteine in silicosis, this study used clinical experiments to verify and analyze the relationship between TNF- $\alpha$, IL- 6 and the clinical therapeutic effect, so as to provide guidance for clinical judgement of the prognosis of silicosis patients. 
Table I. General information.

\begin{tabular}{|c|c|c|c|c|}
\hline Features & $\begin{array}{l}\text { Control group } \\
\quad(\mathrm{n}=108)\end{array}$ & $\begin{array}{l}\text { Observation group } \\
\qquad(\mathrm{n}=88)\end{array}$ & $\chi^{2} / \mathrm{t}$ & P-value \\
\hline $\operatorname{Sex}[\mathrm{n}(\%)]$ & & & 0.444 & 0.505 \\
\hline Male & $70(64.81)$ & $61(69.32)$ & & \\
\hline Female & $38(35.19)$ & $27(30.68)$ & & \\
\hline Age (years) & $48.67 \pm 12.59$ & $52.44 \pm 14.72$ & 1.932 & 0.055 \\
\hline $\operatorname{BMI}\left(\mathrm{kg} / \mathrm{m}^{2}\right)$ & $18.25 \pm 0.83$ & $18.37 \pm 0.62$ & 1.124 & 0.262 \\
\hline Stage $[\mathrm{n}(\%)]$ & & & 0.345 & 0.833 \\
\hline I & $55(50.92)$ & $41(46.59)$ & & \\
\hline II & $35(32.41)$ & $31(35.23)$ & & \\
\hline III & $18(16.67)$ & $16(18.18)$ & & \\
\hline Dust exposure time (years) & $7.43 \pm 3.25$ & $7.83 \pm 3.47$ & & \\
\hline Type of work [n (\%)] & & & 4.361 & 0.499 \\
\hline Roadheader & $32(29.63)$ & $23(26.14)$ & & \\
\hline Stonecutter & $39(36.11)$ & $26(29.55)$ & & \\
\hline Raw material worker & $11(10.18)$ & $12(13.64)$ & & \\
\hline Rock driller & $13(12.04)$ & $12(13.64)$ & & \\
\hline Stoker & $11(10.18)$ & $9(10.23)$ & & \\
\hline Comminution worker & $2(1.85)$ & $6(6.82)$ & & \\
\hline Smoking history [n (\%)] & & & 0.034 & 0.854 \\
\hline Yes & $22(20.37)$ & $17(19.32)$ & & \\
\hline No & $86(79.63)$ & $71(80.68)$ & & \\
\hline
\end{tabular}

\section{Patients and methods}

General materials. In this study, 196 patients with silicosis admitted to Qingdao Central Hospital (Qingdao, China), aged 40 to 70 years, were collected by prospective analysis. The patients were divided into two groups according to the different methods of treatment. One hundred and eight patients in the control group received routine treatment, including anti-inflammatory, cough and asthma relief. Eightyeight patients in the observation group were treated with tetrandrine combined with $\mathrm{N}$-acetylcysteine on the basis of routine treatment.

\section{Inclusion and exclusion criteria}

Inclusion criteria: All patients met the 2015 GBZ70-2015 Diagnostic Criteria for Pneumoconiosis (11); the patient was treated for the first time in this hospital; the patients were not exposed to silicon dioxide during treatment; this study followed the principles of the Helsinki Declaration.

Exclusion criteria: Patients with history of tuberculosis or period of tuberculosis activity; patients with other causes of pulmonary fibrosis; patients with lung tumor; patients with other severe organ dysfunction; patients with autoimmune diseases and history of it; patients with systemic inflammatory response syndrome; pregnant or lactating women.

The study was approved by the Ethics Committee of Qingdao Central Hospital, and patients were consulted by telephone or letter. Each patient and/or their family signed an informed consent form.

\section{Therapeutic method}

Control group.Included anti-inflammatory, cough and asthma relief; the main drugs were ambroxol hydrochloride oral liquid (Harbin Renhuang Pharmaceutical Co., Ltd.; SFDA Approval no. H20067008) $10 \mathrm{ml} /$ time, twice a day, tiobutramine bromide powder inhaler (Jiangsu Zhengda Tianqing Pharmaceutical Co., Ltd.; SFDA Approval no. H20060454), one each time, once a day, with nutritional support and lung rehabilitation training.

Observation group. On the basis of the control group, tetrandrine combined with $\mathrm{N}$-acetylcysteine was given. Tetrandrine (Zhejiang China Resources Sanjiu Zhongyi Pharmaceutical; SFDA Approval no. 2236H1330) 60 mg-100 mg/time, 3 times a day, taken for 6 days and then stopped for 1 day, with a course of treatment of 3 months. Acetylcysteine effervescent tablets (Zambon S.p.A.; SFDA Approval no. H20140449) 1 tablet/time, 1-2 times/day. The treatment lasted 8 months in both groups.

Evaluation criteria. The curative effect of the two groups was analyzed by X-ray, and the incidence of adverse reactions was compared between the two groups. The changes of pulmonary function indexes and the distance of 6-min walking test were analyzed in the groups. The changes of IL- 6 and TNF- $\alpha$ in the peripheral blood of the two groups were measured before and after treatment, and the relationship between IL-6, TNF- $\alpha$ and the therapeutic effect was analyzed.

Test method. Efficacy assessment methods: TOSHIBA multi-function remote-control digital X-ray machine was 
Table II. Analysis of clinical efficacy [n (\%)].

\begin{tabular}{lccrr}
\hline Clinical efficacy & $\begin{array}{c}\text { Control group } \\
(\mathrm{n}=108)\end{array}$ & $\begin{array}{c}\text { Observation group } \\
(\mathrm{n}=88)\end{array}$ & $\chi^{2}$ & P-value \\
\hline Markedly effective & $49(45.37)$ & $58(65.91)$ & 8.252 & 0.004 \\
Effective & $36(33.33)$ & $28(31.82)$ & 0.051 & 0.822 \\
Ineffective & $23(21.30)$ & $2(2.27)$ & 57.843 & $<0.001$ \\
Total effective rate & $85(78.70)$ & $86(97.73)$ & 57.843 & $<0.001$ \\
\hline
\end{tabular}

Table III. Analysis of the incidence of the complications [n (\%)].

\begin{tabular}{lcccr}
\hline Complications & $\begin{array}{c}\text { Control group } \\
(\mathrm{n}=108)\end{array}$ & $\begin{array}{c}\text { Observation group } \\
(\mathrm{n}=88)\end{array}$ & $\chi^{2}$ & P-value \\
\hline Abdominal distension & $3(2.78)$ & $2(2.27)$ & 0.050 & 0.824 \\
Nausea & $2(1.85)$ & $3(3.41)$ & 0.473 & 0.492 \\
Chromatosis & $0(0.00)$ & $2(2.27)$ & Fisher & 0.200 \\
Itching of the skin & $2(1.85)$ & $1(1.14)$ & 0.165 & 0.685 \\
Transaminase elevation & $0(0.00)$ & $3(3.41)$ & Fisher & 0.088 \\
Total incidence rate of adverse reactions & $7(6.48)$ & $11(12.50)$ & 2.106 & 0.147 \\
\hline
\end{tabular}

purchased from Toshiba Medical Systems Co., Ltd.; all the patients were examined by X-ray before and after treatment, and the films were read by 3 attending doctors with 10-year working experience in this hospital. Grading: the lung texture is obviously reduced to be markedly effective, the lung shadow is reduced to be effective, the lung texture is not changed or even increased and aggravated in ineffective result. Total effective rate $=$ markedly effective rate + effective rate.

Lung function: MSPFT- B pulmonary function instrument was purchased from Shanghai Hanfei Medical Devices Co., Ltd.; main observation indexes were respiratory rate (RR), forced vital capacity (FVC), and first second forced expiratory volume (FEV1).

6-min walk experiment: A $20 \mathrm{~m}$-long straight line was plotted on the flat to record the patient's 6-min walking distance and assess the patient's exercise tolerance.

ELISA: Levels of IL-6, TNF- $\alpha$ were detected by ELISA; the test kits were purchased from Wuhan Ellette Biotechnology Co., Ltd. (SFDA Approval no. E-EL-H0102c, E-EL-H0109c), and the specific test steps referred to the kit instructions.

Statistical analysis. SPSS 19.0 (Asia Analytics Formerly SPSS China) was used. Enumeration data were expressed in rates. $\chi^{2}$ test was used to compare the rate. Measurement data were expressed in mean \pm SD. Independent sample t-test was used for comparison between the two groups. Paired t-test was used for intra-group comparison. The relationship between IL-6, TNF- $\alpha$ and the therapeutic effect was analyzed by ROC curve. $\mathrm{P}<0.05$ was considered a statistically significant difference.

\section{Results}

General materials. There were 108 patients in the control group, including 70 males $(64.81 \%)$ and 38 females $(35.19 \%)$ aged $48.67 \pm 12.59$ years. There were 88 patients in the observation group, including 61 males $(69.32 \%)$ and 27 females (30.68\%) aged $52.44 \pm 14.72$ years. There was no significant difference in sex ratio and age between the two groups $(\mathrm{P}>0.05)$. There was no significant difference in BMI, stage, dust exposure time and type of work between the two groups (P>0.05) (Table I).

Analysis of clinical efficacy. The markedly effective rate and total effective rate of treatment in the observation group were significantly higher than those in the control group $(\mathrm{P}<0.05)$, and the ineffective rate was lower than that in the control group $(\mathrm{P}<0.05)$. There was no significant difference in the effective rate between the two groups $(\mathrm{P}>0.05)$ (Table II).

Incidence of complications. In the control group, complications (abdominal distension, nausea, itching of the skin) occurred in 7 cases. In the observation group, complications (abdominal distension, nausea, skin rashes, itching of the skin and transaminase elevation) occurred in 11 cases, and disappeared after withdrawal. There was no significant difference in the incidence of complications between the two groups ( $\mathrm{P}>0.05)$ (Table III).

Lung function. There was no significant difference in RR, FVC and FEV1 between the two groups before treatment $(\mathrm{P}>0.05)$. After treatment, RR decreased, whereas, FVC and FEV1 increased in both groups $(\mathrm{P}<0.05)$, and $\mathrm{RR}$ in the observation group was lower than that in the control group $(\mathrm{P}<0.05), \mathrm{FVC}$ and FEV1 were higher than those in control group $(\mathrm{P}<0.05)$ (Table IV).

6-min walk experiment. There was no significant difference in the results of 6-min walking test between the two groups 
Table IV. Detection of lung function.

\begin{tabular}{|c|c|c|c|c|}
\hline Lung function index & $\begin{array}{l}\text { Control group } \\
\quad(n=108)\end{array}$ & $\begin{array}{l}\text { Observation group } \\
\qquad(\mathrm{n}=88)\end{array}$ & $\mathrm{t}$ & P-value \\
\hline \multicolumn{5}{|l|}{ RR (time/min) } \\
\hline Before treatment & $42.31 \pm 7.15$ & $43.26 \pm 7.64$ & 0.897 & 0.371 \\
\hline After treatment & $37.72 \pm 5.42^{\mathrm{a}}$ & $30.15 \pm 5.13^{\mathrm{a}}$ & 9.961 & $<0.001$ \\
\hline \multicolumn{5}{|l|}{$\mathrm{FVC}(\%)$} \\
\hline Before treatment & $75.16 \pm 7.57$ & $76.21 \pm 7.42$ & 0.975 & 0.331 \\
\hline After treatment & $80.63 \pm 7.28^{\mathrm{a}}$ & $89.06 \pm 7.17^{\mathrm{a}}$ & 8.118 & $<0.001$ \\
\hline \multicolumn{5}{|l|}{ FEV1 (\%) } \\
\hline Before treatment & $71.42 \pm 8.04$ & $70.58 \pm 8.26$ & 0.719 & 0.473 \\
\hline After treatment & $74.33 \pm 7.53^{\mathrm{a}}$ & $85.67 \pm 7.85^{\mathrm{a}}$ & 10.288 & $<0.001$ \\
\hline
\end{tabular}

${ }^{\mathrm{a}} \mathrm{P}<0.05$, compared with the same group before treatment. RR, respiratory rate; FVC, forced vital capacity; FEV1, first second forced expiratory volume.

Table V. Changes of IL-6 and TNF- $\alpha$ expression levels.

\begin{tabular}{lccr}
\hline Expression levels & $\begin{array}{c}\text { Control group } \\
(\mathrm{n}=108)\end{array}$ & $\begin{array}{c}\text { Observation group } \\
(\mathrm{n}=88)\end{array}$ & $\mathrm{t}$ \\
\hline IL-6 $(\mathrm{ng} / \mathrm{ml})$ & & & 0.588 \\
Before treatment & $15.39 \pm 6.83$ & $15.97 \pm 8.14$ & 4.388 \\
After treatment & $13.32 \pm 5.35^{\mathrm{a}}$ & $10.01 \pm 5.13^{\mathrm{a}}$ & 0.001 \\
TNF- $\alpha$ (ng/ml) & & & 1.291 \\
Before treatment & $21.59 \pm 5.59$ & $20.22 \pm 9.13$ & 3.178 \\
After treatment & $19.75 \pm 6.34^{\mathrm{a}}$ & $17.11 \pm 5.02^{\mathrm{a}}$ & 0.198 \\
\hline
\end{tabular}

${ }^{\mathrm{a}} \mathrm{P}<0.05$, compared with the same group before treatment. IL-6, interleukin-6; TNF- $\alpha$, tumor necrosis factor- $\alpha$.

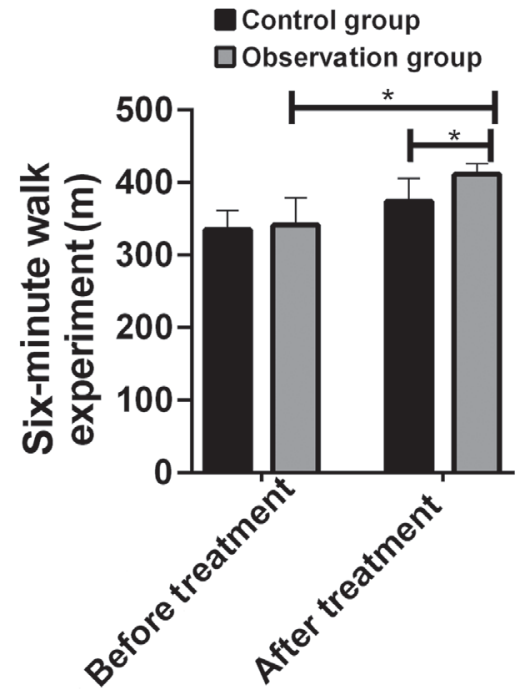

Figure 1. Results of the 6-min walk experiment. After treatment, the 6-min walking distance in the observation group was significantly longer than that in the control group. ${ }^{*} \mathrm{P}<0.05$.

before treatment $(\mathrm{P}>0.05)$, but the 6-min walking distance of the two groups increased after treatment $(\mathrm{P}<0.05)$, and the 6-min walking distance in the observation group was longer than that in the control group $(\mathrm{P}<0.05)$ (Fig. 1).

Changes of IL- 6 and TNF- $\alpha$ expression levels. There was no significant difference in serum IL- 6 and TNF- $\alpha$ levels between the two groups before treatment $(\mathrm{P}>0.05)$. After treatment, the levels of IL- 6 and TNF- $\alpha$ in the two groups decreased $(\mathrm{P}<0.05)$, and the IL-6 and TNF- $\alpha$ levels in the observation group after treatment were significantly lower than those in the control group $(\mathrm{P}<0.05)$ (Table $\mathrm{V})$.

Relationship between IL-6,TNF- $\alpha$ and the clinical therapeutic effect. According to the effect of clinical treatment, the patients were re-divided into two groups. The patients who had significant therapeutic effect and the effective treatment were treated as the effective group, and those who had no effect were treated as the ineffective group. The results of ROC curve analysis showed that IL- 6 evaluation of effective and ineffective AUC, critical value, sensitivity and specificity were $0.743,13.72 \mathrm{ng} / \mathrm{ml}, 52.42$ and $65.38 \%$, respectively. The AUC, critical value, sensitivity and specificity of TNF- $\alpha$ were $0.723,17.74 \mathrm{ng} / \mathrm{ml}, 92.00$ and $49.71 \%$, respectively (Table VI and Fig. 2). 
Table VI. Relationship between IL-6, TNF- $\alpha$ and the clinical therapeutic effect.

\begin{tabular}{lcc}
\hline ROC curve index & IL-6 & TNF- $\alpha$ \\
\hline AUC & 0.743 & 0.723 \\
$95 \% \mathrm{Cl}$ & $0.635-0.850$ & $0.637-0.809$ \\
Cut-off & $13.72 \mathrm{ng} / \mathrm{ml}$ & $17.74 \mathrm{ng} / \mathrm{ml}$ \\
Sensitivity (\%) & 52.42 & 92.00 \\
Specificity (\%) & 65.38 & 49.71 \\
\hline
\end{tabular}

IL-6, interleukin-6; TNF- $\alpha$, tumor necrosis factor- $\alpha$.

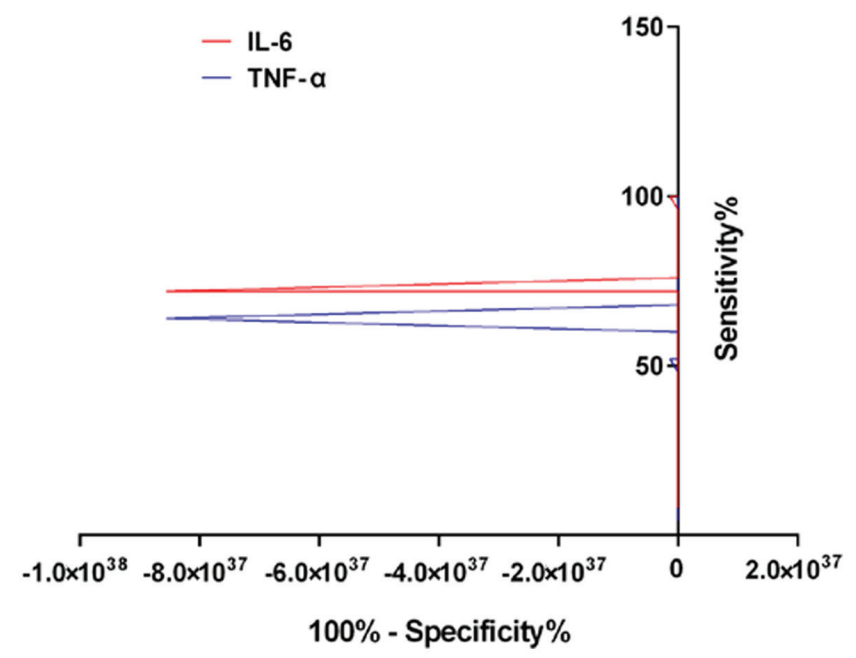

Figure 2. Clinical value of IL-6 and TNF- $\alpha$ in the treatment of silicosis IL-6 evaluation of effective and ineffective AUC, critical value, sensitivity and specificity were $0.743,13.72 \mathrm{ng} / \mathrm{ml}, 52.42$ and $65.38 \%$, respectively. The AUC, critical value, sensitivity and specificity of TNF- $\alpha$ were 0.723 , $17.74 \mathrm{ng} / \mathrm{ml}, 92.00$ and $49.71 \%$, respectively.

\section{Discussion}

Silicosis still has a high incidence in many countries in the world, including China, and South Africa. The main pathogenesis of silicosis is the humoral immune excess caused by macrophages, lymphocytes and their cytokines, as well as cellular immunosuppression, which leads to immune dysfunction or imbalance. Inflammatory reaction and pulmonary fibrosis are important pathological changes and cause respiratory impairment (12). At present, there is no cure method for silicosis. It is of great significance for clinical treatment of silicosis to find new drugs or therapeutic regimens.

The anti-inflammatory effect of tetrandrine has been proven. Seow et al (13) reported that tetrandrine can inhibit the uptake of deoxyglucose by neutrophils and monocytes and inhibit the adhesion of inflammatory cells. Mazurek et al (14) demonstrated that tetrandrine could inhibit the random movement, chemotaxis and phagocytosis of human neutrophils in vitro. It can inhibit the shunt activity of hexose-monophosphate and the production of hydrogen peroxide and superoxide anion, so as to exert anti-inflammatory effects such as anti-phagocytosis, and anti-oxidation. Tetrandrine also plays an anti-fibrotic role through calcium channel block. In the past few decades, tetrandrine has been widely used in the clinical treatment of silicosis, and the combination of drugs has a more obvious inhibitory effect on the formation of fibrosis (15). But there is no single drug or treatment that can completely cure silicosis so far. Acetylcysteine is the precursor of glutathione, which can supplement the content of glutathione in the body and enhance the antioxidant effect of the organism. Acetylcysteine also has an antioxidant effect that protects biological macromolecules by reducing oxidized disulfide bonds to eliminate hydroxyl radicals and hydrogen peroxide, and inhibits the proliferation of fibroblasts and the formation of extracellular matrix to play an anti-fibrotic role $(16,17)$. Acetylcysteine has been used in the treatment of pulmonary fibrosis in the past, such as the treatment of idiopathic pulmonary fibrosis with pirfenidone, and has achieved a good clinical therapeutic effect (18). However, few studies have been reported on the application of acetylcysteine in silicosis, especially in clinical studies.

The clinical efficacy of acetylcysteine combined with tetrandrine tablets in the treatment of silicosis was studied prospectively. There was no statistical difference in sex, age and other general data between the two groups, suggesting that the two groups of patients included in this study were comparable. We first analyzed the therapeutic effects of the two groups of patients. Compared with the conventional treatment, the total effective rate and markedly effective rate of treatment for silicosis patients were significantly increased with the addition of acetylcysteine and tetrandrine. These results suggest that acetylcysteine combined with tetrandrine can effectively improve the therapeutic effect of silicosis. Some indexes of pulmonary function and the results of 6-min walking test also suggested the positive effect of acetylcysteine and tetrandrine. But we calculated the incidence of adverse reactions in the two groups of patients to avoid more adverse reactions caused by increased use of drugs. The results showed that there was no significant difference in the incidence of total adverse reactions and single adverse reaction between the two groups, which suggested that increasing the use of tetrandrine and acetylcysteine was still safe. But the patients in the observation group had two new adverse reactions, rash and elevation of transaminase. Due to the long period of clinical treatment of silicosis, it is necessary to remind patients to pay close attention to the changes in the course of treatment, and to go to the hospital for regularly review. At present, there are few studies on the combination of acetylcysteine and tetrandrine in the treatment of silicosis. Some studies reported that, compared to routine treatment, tetrandrine combined with acetylcysteine can effectively alleviate clinical symptoms such as cough and dyspnea, improve pulmonary function, reduce oxidative stress levels and limit the progression of fibrosis in silicosis patients, which also confirms the accuracy of our results.

This study did not analyze the changes of oxidative stress levels in the two groups of patients, but analyzed the changes of IL-6 and TNF- $\alpha$ and its relationship with therapeutic effect. IL- 6 and TNF- $\alpha$ are two factors closely related to inflammatory reaction, and their elevated levels may indicate an increase in the degree of inflammatory response in the body $(19,20)$. The results of this study showed that the degree of improvement of inflammatory reaction in the observation group was significantly better than that in the control group. The results of ROC analysis suggest that the levels of IL- 6 and TNF- $\alpha$ have a good 
diagnostic value in the clinical treatment of silicosis. The AUC of effective and ineffective differential therapy were 0.743 and 0.723 , respectively. These results suggest that the detection of IL- 6 and TNF- $\alpha$ may reduce the use of X-ray and other imaging methods to a certain extent, and reduce the cost of treatment.

This study also has some shortcomings. The prospective study inevitably has Hawthorne effect. In addition, this study did not carry out a long follow-up, so the final outcome of the patient cannot be judged. We will continue to track patients and evaluate their long-term outcomes.

In conclusion, tetrandrine combined with acetylcysteine can effectively improve the clinical therapeutic effect of silicosis, alleviate the degree of inflammation and improve the levels of IL-6 and TNF- $\alpha$ in peripheral blood, which is valuable in judging the clinical therapeutic effect of silicosis.

\section{Acknowledgements}

Not applicable.

\section{Funding}

This study was supported by Qingdao Medical Scientific Research Guidance Plan for 2014 (no. 2014-WJZD076).

\section{Availability of data and materials}

The datasets used and/or analyzed during the present study are available from the corresponding author on reasonable request.

\section{Authors' contributions}

JS and PS analyzed and interpreted the patient general data. YW performed ELISA. YC was responsible for analysis of observation indicators. JS wrote the manuscript. All the authors read and approved the final manuscript.

\section{Ethics approval and consent to participate}

The study was approved by the Ethics Committee of Qingdao Central Hospital (Qingdao, China). Patients who participated in this study, had complete clinical data. Patients and/or their families signed an informed consent.

\section{Patient consent for publication}

Not applicable.

\section{Competing interests}

The authors declare that they have no competing interests.

\section{References}

1. Lee S, Hayashi H, Mastuzaki H, Kumagai-Takei N, Otsuki T: Silicosis and autoimmunity. Curr Opin Allergy Clin Immunol 17: 78-84, 2017.

2. Yew WW, Leung CC, Chang KC, Zhang Y, Chan DP: Can treatment outcomes of latent TB infection and TB in silicosis be improved? J Thorac Dis 11: E8-E10, 2019.
3. Bang KM, Mazurek JM, Wood JM, White GE, Hendricks SA, Weston A, Centers for Disease Control and Prevention (CDC): Silicosis mortality trends and new exposures to respirable crystalline silica - United States, 2001-2010. MMWR Morb Mortal Wkly Rep 64: 117-120, 2015.

4. Brown T, Darnton A, Fortunato L and Rushton L; British Occupational Cancer Burden Study Group: Therapeutic effects of adipose-tissue-derived mesenchymal stromal cells and their extracellular vesicles in experimental silicosis. Br J Cancer 107: S56-S70, 2012.

5. Field RW, Withers BL: Occupational and environmental causes of lung cancer. Clin Chest Med 33: 681-703, 2012.

6. Bhagya N, Chandrashekar KR: Tetrandrine - a molecule of wide bioactivity. Phy tochemistry 125: 5-13, 2016.

7. Zhao YZ, Kim JY, Park EJ, Lee SH, Woo SW, Ko G, Sohn DH: Tetrandrine induces apoptosis in hepatic stellate cells. Phytother Res 18: 306-309, 2004.

8. Zhang L, He YL, Li QZ, Hao XH, Zhang ZF, Yuan JX, Bai YP, Jin YL, Liu N, et al: N-acetylcysteine alleviated silica-induced lung fibrosis in rats by down-regulation of ROS and mitochondrial apoptosis signaling. Toxicol Mech Methods 24: 212-219, 2014.

9. Chen Z, Zhao L, Zhao F, Yang G and Wang JJ: Tetrandrine suppresses lung cancer growth and induces apoptosis, potentially via the VEGF/HIF-1 $\alpha /$ ICAM-1 signaling pathway. Oncol Lett 15: 7433-7437, 2018.

10. Xiao Y, Xia H, Zhu L, Li X, Chen R, Yin X, Jiang Z, Feng L, Chen J, et al: Study on the therapeutic effects of tetrandrine combined with $\mathrm{N}$-acetylcysteine on experimental silicosis of rats. Zhonghua Lao Dong Wei Sheng Zhi Ye Bing Za Zhi 33: 519-522, 2015.

11. Zhou Z: Understanding the administrative regulation on occupational health and trend in China. J Occup Health 60: 126-131, 2017.

12. Maeda M, Nishimura Y, Kumagai N, Hayashi H, Hatayama T, Katoh M, Miyahara N, Yamamoto S, Hirastuka J, et al: Dysregulation of the immune system caused by silica and asbestos. J Immunotoxicol 7: 268-278, 2010.

13. Seow WK, Si-Ying L and Thong YH: Inhibitory effects of tetrandrine on human neutrophil and monocyte adherence, Immunol Lett 13: 83-88, 1986.

14. Mazurek JM, Wood JM, Schleiff PL, Weissman DN: Surveillance for silicosis deaths among persons aged 15-44 years - United States, 1999-2015. MMWR Morb Mortal Wkly Rep 66: 747-752, 2017.

15. Xie QM, Tang HF, Chen JQ and Bian RL: Pharmacological actions of tetrandrine in inflammatory pulmonary diseases. Acta Pharmacol Sin 23: 1107-1113, 2002.

16. Monti D, Sotgia F, Whitaker-Menezes D, Tuluc M, Birbe R, Berger A, Lazar M, Cotzia P, Draganova-Tacheva R, et al: Pilot study demonstrating metabolic and anti-proliferative effects of in vivo anti-oxidant supplementation with $\mathrm{N}$-acetylcysteine in Breast Cancer. Semin Oncol 44: 226-232, 2017.

17. Mazurek JM, Schleiff PL, Wood JM, Hendricks SA, Weston A; Centers for Disease Control and Prevention (CDC): Notes from the Field: Update: Silicosis Mortality - United States, 1999-2013. MMWR Morb Mortal Wkly Rep 64: 653-654, 2015.

18. Sakamoto S, Muramatsu Y, Satoh K, Ishida F, Kikuchi N, Sano G, Sugino K, Isobe K, Takai Y, et al: Effectiveness of combined therapy with pirfenidone and inhaled $\mathrm{N}$-acetylcysteine for advanced idiopathic pulmonary fibrosis: A case-control study. Respirology 20: 445-452, 2015.

19. Del Giudice M, Gangestad S W: Rethinking IL-6 and CRP: Why they are more than inflammatory biomarkers, and why it matters. Brain Behav Immun 70: 61-75, 2018.

20. Charrad R, Berraïes A, Hamdi B, Ammar J, Hamzaoui K and Hamzaoui A: Anti-inflammatory activity of IL-37 in asthmatic children: Correlation with inflammatory cytokines TNF- $\alpha$, IL- $\beta$, IL-6 and IL-17A. Immunobiology 221: 182-187, 2015.

This work is licensed under a Creative Commons Attribution-NonCommercial-NoDerivatives 4.0 International (CC BY-NC-ND 4.0) License. 